\title{
A Point Model for the Free Cyclic Submodules over Ternions
}

\author{
Hans Havlicek, Jarosław Kosiorek and Boris Odehnal
}

\begin{abstract}
We show that the set of all (unimodular and non-unimodular) free cyclic submodules of $T^{2}$, where $T$ is the ring of ternions over a commutative field, admits a point model in terms of a smooth algebraic variety.
\end{abstract}

Mathematics Subject Classification. 51B99, 51C99, 14J26, 16D40.

Keywords. Ternions, projective line, cyclic submodules, Grassmann variety, Segre variety.

\section{Introduction}

The present paper is devoted to the study of the geometry of free cyclic submodules of $T^{2}$, where $T$ denotes the ring of ternions (upper triangular $2 \times 2$ matrices) over a commutative field $\mathbb{F}$. In a more geometric language we refer to these submodules as ternionic points. We show that the set of all such points admits a model in terms of a smooth algebraic variety in a projective space on an 8-dimensional vector space over $\mathbb{F}$. Our exposition is based on the Grassmann variety representing the planes (3-dimensional subspaces) of $\mathbb{F}^{6}$ and the recent paper [8], where a model for the set of ternionic points in terms of planes of $\mathbb{F}^{6}$ was given and exhibited.

From [9], the set of ternionic points splits into two orbits under the natural action of $\mathrm{GL}_{2}(T)$. One orbit comprises the set of unimodular points, the elements of the other orbit are called non-unimodular points. The variety representing the entire set of ternionic points accordingly splits into two parts. The first arises by removing a single line from the variety, i.e., we obtain a quasiprojective variety in the terminology of [15]. The second part is just that distinguished line. So, as regards the unimodular points, our results parallel 
those of A. Herzer, who developed a very general representation theory (for unimodular points only). See [5, Chapt. 11 and 12] and [10] for further details. It seems worth pointing out that all points of the distinguished line are smooth, thus giving a negative answer to the question whether non-unimodularity of a ternionic point would imply its image point being singular.

The geometry over ternions based on unimodular points has attracted many authors. We refer to $[1-4,6,7]$, and the references therein. There are but a few papers dealing with the properties of the remaining (non-unimodular) ternionic points $[8,9,13,14]$.

Results and notions which are used without further reference can be found, for example, in $[5,10]$, and [12].

\section{Main Results}

Let $\mathbb{F}$ be a commutative field and $T$ be the ring of ternions, i.e., the upper triangular matrices

$$
\left(\begin{array}{rr}
a_{11} & a_{12} \\
0 & a_{22}
\end{array}\right)
$$

with entries $a_{i j} \in \mathbb{F}$. Sometimes we identify $x \in \mathbb{F}$ with the ternion $x I$, where $I$ is the $2 \times 2$ identity matrix. $\mathbb{F}$ is the center of $T$ and $T$ is a three-dimensional algebra over $\mathbb{F}$.

According to [9] the non-zero cyclic submodules of the free $T$-left module $T^{2}$ fall into five orbits under the natural action of the group $\mathrm{GL}_{2}(T)$. In the following we focus on two types of submodules given by a representative

$$
X_{0}=T\left[\left(\begin{array}{ll}
1 & 0 \\
0 & 1
\end{array}\right),\left(\begin{array}{ll}
0 & 0 \\
0 & 0
\end{array}\right)\right]=\left\{\left[\left(\begin{array}{ll}
x & y \\
0 & z
\end{array}\right),\left(\begin{array}{ll}
0 & 0 \\
0 & 0
\end{array}\right)\right] \mid x, y, z \in \mathbb{F}\right\}
$$

and

$$
Y_{0}=T\left[\left(\begin{array}{ll}
0 & 0 \\
0 & 1
\end{array}\right),\left(\begin{array}{ll}
0 & 1 \\
0 & 0
\end{array}\right)\right]=\left\{\left[\left(\begin{array}{ll}
0 & y \\
0 & z
\end{array}\right),\left(\begin{array}{ll}
0 & x \\
0 & 0
\end{array}\right)\right] \mid x, y, z \in \mathbb{F}\right\} .
$$

An arbitrary submodule $X$ from the orbit of $X_{0}$ given in (2) is obtained by applying $S \in \mathrm{GL}_{2}(T)$ to $X_{0}$, i.e., $X=X_{0} S$ and will be called $X$-submodule in the following. Submodules of this type are free and arise from unimodular pairs of $T^{2}$. They are the points of the projective line over $T$ as considered in [5] and [10]. This yields the general form of an $X$-submodule as

$$
X=\left\{\left[\left(\begin{array}{cc}
a_{11} x & a_{12} x+a_{22} y \\
0 & a_{22} z
\end{array}\right),\left(\begin{array}{cc}
b_{11} x & b_{12} x+b_{22} y \\
0 & b_{22} z
\end{array}\right)\right] \mid x, y, z \in \mathbb{F}\right\},
$$

with the constraint

$$
\left(a_{11}, b_{11}\right) \neq(0,0) \neq\left(a_{22}, b_{22}\right) .
$$


In the same way we obtain the general form of the $Y$-submodules using (3)

$$
Y=\left\{\left[\left(\begin{array}{cc}
0 & a_{22} y+c_{22} x \\
0 & a_{22} z
\end{array}\right),\left(\begin{array}{cc}
0 & b_{22} y+d_{22} x \\
0 & b_{22} z
\end{array}\right)\right] \mid x, y, z \in \mathbb{F}\right\},
$$

with the additional condition

$$
a_{22} d_{22}-b_{22} c_{22} \neq 0 \text {. }
$$

The $Y$-submodules are precisely those free cyclic submodules which cannot be generated by a unimodular pair. We call them free non-unimodular points.

We identify $T^{2}$ with $\mathbb{F}^{6}$ by

$$
\left(\begin{array}{rr|rr}
a_{11} & a_{12} & b_{11} & b_{12} \\
0 & a_{22} & 0 & b_{22}
\end{array}\right) \mapsto\left(a_{11}, b_{11}, a_{22}, b_{22}, a_{12}, b_{12}\right) \in \mathbb{F}^{6} .
$$

From (4) we see that the $X$-submodules are three-dimensional subspaces of $\mathbb{F}^{6}$ and we can extract the base vectors

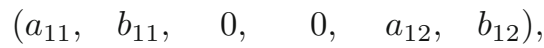

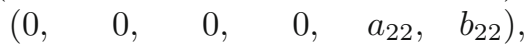

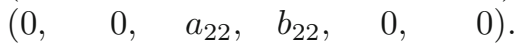

In the following we use the Grassmann variety $\mathcal{G} \subset \mathbb{F}^{20}=\mathbb{F}^{6} \wedge \mathbb{F}^{6} \wedge \mathbb{F}^{6}$ representing the three-dimensional subspaces of $\mathbb{F}^{6}$. We denote the vectors of the standard basis of $\mathbb{F}^{20}$ by $e_{i j k}, 1 \leq i<j<k \leq 6$, and write $E_{i j k}=\mathbb{F} e_{i j k}$ for the respective base point of the projective coordinate system.

Every $X$-submodule is now represented by a point in the Grassmann variety $\mathcal{G}$. We compute the Grassmann coordinates of an $X$-submodule with base points (8) and the non-vanishing coordinates read

$$
\begin{aligned}
& p_{135}=-a_{11} a_{22}^{2}, \quad p_{136}=p_{145}=-a_{11} a_{22} b_{22}, \quad p_{146}=-a_{11} b_{22}^{2}, \\
& p_{235}=-b_{11} a_{22}^{2}, \quad p_{236}=p_{245}=-b_{11} a_{22} b_{22}, \quad p_{246}=-b_{11} b_{22}^{2} \text {, } \\
& p_{356}=a_{22}\left(a_{12} b_{22}-b_{12} a_{22}\right), \\
& p_{456}=b_{22}\left(a_{12} b_{22}-b_{12} a_{22}\right) .
\end{aligned}
$$

This gives a parametric representation of the Grassmann image $\mathcal{X}$, say, of the set of $X$-submodules. The parameters $a_{11}, a_{22}, b_{11}, b_{22} \in \mathbb{F}$ in $(9)$ are subject to the restrictions given in (5), but there is no restriction on $a_{12}$ and $b_{12}$. An easy calculation shows that the Grassmann image $\mathcal{Y}$ of the set comprising all $Y$-submodules is the line spanned by $E_{356}$ and $E_{456}$. A parametric representation of $\mathcal{Y}$ is given by

$$
p_{356}=a_{22}\left(a_{22} d_{22}-b_{22} c_{22}\right) \quad \text { and } \quad p_{456}=b_{22}\left(a_{22} d_{22}-b_{22} c_{22}\right),
$$

where again only the non-vanishing coordinate functions are given.

We want to understand the set $\mathcal{X} \cup \mathcal{Y}$. For that end we derive the equations of $\mathcal{X} \cup \mathcal{Y}$ and show the geometric meaning of both, its parametrization and the equations as well. 
At first we observe that $\mathcal{X} \cup \mathcal{Y}$ is contained in a subspace of dimension 8 which is given by the linear equations

$$
\begin{gathered}
p_{123}=p_{124}=p_{125}=p_{126}=p_{134}=p_{156}=p_{234}=p_{256}=p_{345}=p_{346}=0, \\
p_{236}-p_{245}=0, \quad p_{136}-p_{145}=0
\end{gathered}
$$

These are precisely the vanishing coordinate functions not mentioned in (9) together with two obvious identities.

In order to describe $\mathcal{X} \cup \mathcal{Y}$ we show:

Lemma 1. For any $(u, v) \in \mathbb{F}^{2} \backslash\{(0,0)\}$ and $i \in\{1,2\}$ let

$$
\begin{aligned}
q_{i}(u, v) & =u^{2} e_{i 35}+u v\left(e_{i 36}+e_{i 45}\right)+v^{2} e_{i 46}, \\
r(u, v) & =u e_{356}+v e_{456} .
\end{aligned}
$$

Then $\gamma(u, v):=\mathbb{F} q_{1}(u, v)+\mathbb{F} q_{2}(u, v)+\mathbb{F} r(u, v)$ is a plane. As $(u, v)$ varies in $\mathbb{F}^{2} \backslash\{(0,0)\}$ the union of these planes equals $\mathcal{X} \cup \mathcal{Y}$.

Proof. First we note that for $i \in\{1,2\}$ and variable $(u, v)$ the points $\mathbb{F} q_{i}(u, v)$ comprise a conic section $c_{i}$ in the plane spanned by $E_{i 35}, \mathbb{F}\left(e_{i 36}+e_{i 45}\right)$, and $E_{i 46}$, whereas the points $\mathbb{F} r(u, v)$ form the line spanned by $E_{356}$ and $E_{456}$. Hence any $\gamma(u, v)$ is a plane.

Given any point of $\mathcal{X}$ with parameters as in (9) and (5), we let $u=a_{22}$ and $v=b_{22}$. Then one immediately reads off from (9) that this point belongs to $\gamma(u, v)$.

Conversely, let $G$ be a point of $\gamma(u, v)$ which lies off the line $\mathcal{Y}$. So we may assume $G=\mathbb{F} g$, where

$$
g=g_{1} q_{1}(u, v)+g_{2} q_{2}(u, v)+g_{3} r(u, v)
$$

with $\left(g_{1}, g_{2}, g_{3}\right) \in \mathbb{F}^{3}$ and $\left(g_{1}, g_{2}\right) \neq(0,0)$. In order to show that the coordinates of $G$ can be expressed as in (9) it suffices to let $a_{22}=u, b_{22}=v, a_{11}=$ $-g_{1}, b_{11}=-g_{2}$. Moreover, since $\left(a_{22}, b_{22}\right) \neq(0,0)$ there exists at least one pair $\left(a_{12}, b_{12}\right) \in \mathbb{F}^{2}$ such that $a_{12} b_{22}-b_{12} a_{22}=g_{3}$.

We already know from (10) that $\mathcal{Y}$ is the Grassmann image of the set of all $Y$-modules.

The parametric representation of $\mathcal{X}$ and $\mathcal{Y}$ from (9) and (10) allows us to derive equations of $\mathcal{X} \cup \mathcal{Y}$ by eliminating parameters. From now on we restrict ourselves to the 8-dimensional subspace given by Eqs. (11) and disregard the ambient 20-dimensional space. In other words, Eqs. (11) are always assumed to be satisfied without further notice. We find nine quadratic equations which will be arranged in three groups. The first two equations

$$
p_{135} p_{146}-p_{145}^{2}=0, \quad p_{235} p_{246}-p_{245}^{2}=0
$$

describe quadratic cones erected on the conic sections $c_{i}$ mentioned in the proof of Lemma 1. These quadratic cones have five-dimensional subspaces for their vertices and six-dimensional generators. 
Further we consider the quadratic equations

$$
\begin{gathered}
p_{146} p_{245}-p_{145} p_{246}=0, \quad p_{135} p_{246}-p_{235} p_{146}=0 \\
p_{135} p_{245}-p_{145} p_{235}=0
\end{gathered}
$$

They determine three quadratic cones on ruled quadrics. Together with the linear equations $p_{356}=p_{456}=0$ the equations (15) describe a Segre variety $\mathcal{S}$ which is the product of a line and a plane, cf. [11, p. 189]. In parametric form this Segre variety can be written as the set of all points with coordinates

$$
p_{i 35}=u_{1} v_{i}, \quad p_{i 36}=p_{i 45}=u_{2} v_{i}, \quad p_{i 46}=u_{3} v_{i}
$$

with $i \in\{1,2\},\left(u_{1}, u_{2}, u_{3}\right) \in \mathbb{F}^{3} \backslash\{(0,0,0)\}$, and $\left(v_{1}, v_{2}\right) \in \mathbb{F}^{2} \backslash\{(0,0)\}$, where all other coordinates are understood to be zero.

Finally the third set of quadratic equations reads

$$
\begin{aligned}
& p_{135} p_{456}-p_{145} p_{356}=0, \quad p_{145} p_{456}-p_{146} p_{356}=0 \\
& p_{235} p_{456}-p_{245} p_{356}=0,
\end{aligned}
$$

These are the equations of quadratic cones on ruled quadrics. All of them have four-dimensional vertices, six-dimensional generators, and share the line $\mathcal{Y}$ spanned by the base points $E_{356}$ and $E_{456}$.

Now we can prove the following result:

Theorem 1. The set $\mathcal{X} \cup \mathcal{Y}$ is a smooth algebraic variety given by the equations (11), (14), (15), and (17).

Proof. It is easily verified that the coordinate functions of both parametric representations, namely that of $\mathcal{X}$ given in (9) and that of $\mathcal{Y}$ given in (10) annihilate Eqs. (11), (14), (15), and (17).

Conversely, we have to show that for any point $P=\mathbb{F}\left(\ldots, p_{i j k}, \ldots\right)$ given by Eqs. (11), (14), (15), and (17) there are parameters such that $P$ can be written as in (9) or (10), respectively.

We distinguish two cases: Assume first that $\left(p_{356}, p_{456}\right) \neq(0,0)$, whereas all other coordinates of $P$ vanish. Then we let $a_{22}=p_{356}$ and $b_{22}=p_{456}$. There exists at least one pair $\left(c_{22}, d_{22}\right) \in \mathbb{F}^{2}$ such that $(7)$ is satisfied. Now (10) shows that $P$ is a point on $\mathcal{Y}$.

Otherwise $\left(p_{135}, p_{145}, p_{146}, p_{235}, p_{245}, p_{246}\right)$ is a non-trivial zero of $(15)$. We infer from (16) that there are parameters $\left(u_{1}, u_{2}, u_{3}\right) \in \mathbb{F}^{3} \backslash\{(0,0,0)\}$ and $\left(v_{1}, v_{2}\right) \in \mathbb{F}^{2} \backslash\{(0,0)\}$ such that

$$
\left(p_{i 35}, p_{i 45}, p_{i 46}\right)=\left(u_{1} v_{i}, u_{2} v_{i}, u_{3} v_{i}\right) \text { for } i \in\{1,2\} .
$$

Hence $\left(p_{i 35}, p_{i 45}, p_{i 46}\right) \neq(0,0,0)$ for at least one value of $i$. We may assume w.l.o.g. that this is the case for $i=1$, whence $v_{1} \neq 0$. We substitute (18) in the first equation of (14), divide by $v_{1}^{2}$, and get the constraint $u_{1} u_{3}-u_{2}^{2}=0$ which 
reminds us of the equation of a conic section. The well-known Veronese parametrization of a conic section shows that there exists a pair $\left(a_{22}, b_{22}\right) \in \mathbb{F}^{2} \backslash\{(0,0)\}$ and a constant $k \in \mathbb{F} \backslash\{0\}$ such that

$$
\left(u_{1}, u_{2}, u_{3}\right)=k\left(a_{22}^{2}, a_{22} b_{22}, b_{22}^{2}\right) .
$$

Further we define $a_{11}:=-k v_{1}, b_{11}:=-k v_{2}$, whence $p_{i 35}, p_{i 45}$, and $p_{i 46}$ for $i \in\{1,2\}$ are already given as in (9). Now we substitute into (17) and obtain

$$
\begin{aligned}
& -a_{11} a_{22}\left(a_{22} p_{456}-b_{22} p_{356}\right)=0, \quad-a_{11} b_{22}\left(a_{22} p_{456}-b_{22} p_{356}\right)=0, \\
& -b_{11} a_{22}\left(a_{22} p_{456}-b_{22} p_{356}\right)=0, \quad-b_{11} b_{22}\left(a_{22} p_{456}-b_{22} p_{356}\right)=0 .
\end{aligned}
$$

At least one of these equations shows us that $\left(p_{356}, p_{456}\right)=m\left(a_{22}, b_{22}\right)$ for some $m \in \mathbb{F}$. As $\left(a_{22}, b_{22}\right) \neq(0,0)$ we can find $a_{12}, b_{12} \in \mathbb{F}$ such that $m=$ $a_{12} b_{22}-b_{12} a_{22}$. Thus, finally, the coordinates of $P$ are expressed like in (9) which shows $P \in \mathcal{X}$.

In order to show that $\mathcal{X} \cup \mathcal{Y}$ is smooth we compute the partial derivatives of the parametrization given in (9). Then it is a simple and straightforward calculation that the subspace spanned by the derivatives is of dimension 4 at any point of $\mathcal{X} \cup \mathcal{Y}$, regardless of the characteristic of $\mathbb{F}$.

\section{Final Remarks}

The contents of Lemma 1 as well as the parametrization of $\mathcal{X}$ given in (9) admit a geometric interpretation. The homogeneous parameter $\left(a_{22}, b_{22}\right) \neq$ $(0,0)$ determines a unique point $\mathbb{F} q_{i}\left(a_{22}, b_{22}\right)$ on either conic section $c_{i}$. Further $\left(a_{22}, b_{22}\right)$ determines a unique point $\mathbb{F r}\left(a_{22}, b_{22}\right)$ on the line $\mathcal{Y}$. Any plane $\gamma$ mentioned in Lemma 1 is spanned by these three points. Thus there is a projective mapping from the projective line of parameters to the planes on $\mathcal{X} \cup \mathcal{Y}$. The homogeneous parameters $\left(a_{12}, b_{12}\right) \neq(0,0)$ and $\left(a_{11}, b_{11}\right) \neq(0,0)$ serve as coordinates of points of $\mathcal{X}$ within the generator planes $\gamma$.

We exhibit some subrings within the ternions. Firstly, for $a_{11}=a_{22}$ and $a_{12}=0$ in (1) we obtain the subring of scalar matrices. It is clearly isomorphic to the ground field $\mathbb{F}$. Accordingly, by letting $a_{11}=a_{22}, b_{11}=b_{22}$, and $a_{12}=b_{12}$ in (9), we obtain a parametric representation of a twisted cubic $\mathcal{F} \subset \mathcal{X}$ as a model for the projective line of $\mathbb{F}$. Note that no $Y$-submodule can be written in terms of scalar matrices.

Secondly, letting $a_{11}=a_{22}$ in (1) we find a representation of the dual numbers over $\mathbb{F}$. As before, no non-unimodular points arise from dual numbers. Hence a parametric representation of the free cyclic submodules is obtained from (9) by substituting $a_{22}=a_{11}$ and $b_{22}=b_{11}$. This yields a ruled surface in a subspace of dimension 6 . Any pair $\left(a_{11}, b_{11}\right) \neq(0,0)$ fixes a point on the line joining the previously mentioned points $C_{1}$ and $C_{2}$. So we have a twisted cubic winding about a "tube-like surface" $\mathcal{T} \subset \mathcal{S}$ whose generators are the lines spanned by corresponding points $C_{1}$ and $C_{2}$. The cubic curve meets any generator of this tube-like surface exactly once. The ruled surface appearing 
as the point model of the free cyclic submodules over the dual numbers has two distinguished directrices: the twisted cubic $\mathcal{F}$ and the line $\mathcal{Y}$. There is a projective correspondence between the directrices, and corresponding points are joined in order to form the generators of this ruled surface.

Thirdly, we put $a_{12}=0$, then formula (1) gives a representation of the double numbers over $\mathbb{F}$. The unimodular points of the projective line over the double numbers form the tube-like surface $\mathcal{T}$ mentioned above. This is easily seen, if we insert $a_{12}=b_{12}=0$ in (9). The surface $\mathcal{T}$ is a ruled surface whose two-dimensional generators appear as the subspaces spanned by corresponding points of $C_{1}$ and $C_{2}$. The surface $\mathcal{T}$ is a subset of the Segre $\mathcal{S}$, for $c_{i}$ is contained in the plane spanned by $\mathbb{F} E_{i 35}, \mathbb{F}\left(e_{i 36}+e_{i 45}\right)$, and $\mathbb{F} E_{i 45}$ with $i \in\{1,2\}$. Non-unimodular points over the double numbers do not exist.

\section{Acknowledgements}

This work was carried out within the framework of the Scientific and Technological Cooperation Poland-Austria 2010-2011. The authors wish to thank Andrzej Matraś (Olsztyn) for his useful remarks in the course of numerous vivid discussions.

Open Access. This article is distributed under the terms of the Creative Commons Attribution License which permits any use, distribution, and reproduction in any medium, provided the original author(s) and the source are credited.

\section{References}

[1] Beck, H.: Eine Cremonasche Raumgeometrie. J. Reine Angew. Math. 175, 129-158 (1936)

[2] Beck, H.: Über Ternionen in der Geometrie. Math. Z. 40(1), 509-520 (1936)

[3] Benz, W.: Über eine Cremonasche Raumgeometrie. Math. Nachr. 80, 225-243 (1977)

[4] Benz, W.: Zur Umkehrung von Matrizen im Bereich der Ternionen. Mitt. Math. Ges. Hambg. 10(7), 509-512 (1979)

[5] Blunck, A., Herzer, A.: Kettengeometrien-Eine Einführung. Shaker Verlag, Aachen (2005)

[6] Depunt, J.: Sur la géométrie ternionienne dans le plan. Bull. Soc. Math. Belg. 11, 123-133 (1959)

[7] Depunt, J.: Grondslagen van de analytische projectieve ternionenmeetkunde van het platte vlak. Verh. Konink. Acad. Wetensch. Lett. Schone Kunst. België. Kl. Wetensch. 22(63) (1960)

[8] Havlicek, H., Matras, A., Pankov, M.: Geometry of free cyclic submodules over ternions. Abh. Math. Semin. Univ. Hambg. 81, 237-249 (2011)

[9] Havlicek, H., Saniga, M.: Vectors, cyclic submodules, and projective spaces linked with ternions. J. Geom. 92(1-2), 79-90 (2009) 
[10] Herzer, A.: Chain geometries. In: Buekenhout, F. (ed.) Handbook of Incidence Geometry, pp. 781-842. Elsevier, Amsterdam (1995)

[11] Hirschfeld, J.W.P., Thas, J.A.: General Galois Geometries. Oxford University Press, Oxford (1991)

[12] Pankov, M.: Grassmannians of Classical Buildings. Algebra and Discrete Mathematics, vol. 2. World Scientific, Singapore (2010)

[13] Saniga, M., Havlicek, H., Planat, M., Pracna, P.: Twin "Fano-snowflakes" over the smallest ring of ternions. SIGMA Symmetry Integrability Geom. Methods Appl. 4, art no. 050 (2008) (electronic)

[14] Saniga, M., Pracna, P.: A Jacobson radical decomposition of the Fano-Snowflake configuration. SIGMA Symmetry Integrability Geom. Methods Appl. 4, art no. 072 (2008) (electronic)

[15] Shafarevich, I.G.: Basic Algebraic Geometry 1. Springer, Berlin (1994)

Hans Havlicek and Boris Odehnal

Institut für Diskrete Mathematik und Geometrie

Technische Universität

Wiedner Hauptstraße 8-10/104

1040 Wien

Austria

e-mail: havlicek@geometrie.tuwien.ac.at;

boris@geometrie.tuwien.ac.at

Jarosław Kosiorek

Faculty of Mathematics and Computer Science

University of Warmia and Mazury

Słoneczna 54

10-710 Olsztyn

Poland

e-mail: kosiorek@matman.uwm.edu.pl

Received: December 7, 2011.

Accepted: April 14, 2012. 